Check for updates

Cite this: Mater. Chem. Front., 2019, 3, 2647

Received 30th April 2019, Accepted 17th July 2019

DOI: $10.1039 / c 9 q m 00279 k$

rsc.li/frontiers-materials

\section{On-site visual discrimination of transgenic food by water-soluble DNA-binding AIEgens $\dagger$}

\author{
Zhe Jiao, ${ }^{\text {ab }}$ Zongning Guo, ${ }^{c}$ Xuelin Huang, ${ }^{c}$ Hongbo Fan, ${ }^{\star a}$ Miao Zhao, ${ }^{d}$ \\ Dianming Zhou, ${ }^{d}$ Xiaolei Ruan, ${ }^{e}$ Pengfei Zhang, iD * ${ }^{f}$ Sixing Zhou ${ }^{a}$ and \\ Ben Zhong Tang iD *b
}

\begin{abstract}
Development of affordable, simple and effective methods for rapid on-site identification of genetically modified organisms (GMO) is of importance due to public concern for transgenic food. In this paper, a visual discrimination method for transgenic food was developed based on a portable polymerase chain reaction (PCR) instrument and water-soluble AIE luminogens (AlEgens). The transgenes were extracted and amplified by the PCR device, further visualized by DNA-binding AIEgens 1,1,2,2-tetrakis[4-(2-bromoethoxy)phenyl] ethene (TTAPE) with a label-free approach. The lighted-up emission could be distinctly identified by the naked eye under a UV lamp or by a portable smartphone. The results showed that only transgenes can be visually detected, which confirmed the specificity of the proposed method. Besides, the whole PCR process was not affected by the addition of AlEgens. The portable system was successfully applied and validated for on-site screening and identification of transgenic papaya, corn and soybean samples. The proposed technique may serve as a general DNA fragment on-site visual discrimination platform for environmental and clinical applications.
\end{abstract}

\section{Introduction}

The debate on the use of genetically modified organisms (GMO) in agriculture and the agro-industry is a public concern involving a combination of scientific, social, and political aspects. There is as yet little unanimity among governments and legislators regarding the foreseeable risks that GMO may pose to human health and the environment. ${ }^{1,2}$ Pressures from consumer groups and public demands have led several countries to require labeling for the presence of GM in foods. In many countries,

\footnotetext{
${ }^{a}$ School of Environment and Civil Engineering, Dongguan University of Technology, Dongguan 523808, China. E-mail: fhb666666@126.com

${ }^{b}$ Department of Chemistry, Hong Kong Branch of Chinese National Engineering Research Centre for Tissue Restoration and Reconstruction, Department of Chemical and Biological Engineering, The Hong Kong University of Science and Technology, Clear Water Bay, Kowloon, Hong Kong. E-mail: tangbenz@ust.hk

${ }^{c}$ Integrated Technology Center of Dongguan Entry-Exit Inspection and Quarantine Bureau, Dongguan 523073, China

${ }^{d}$ Tianjin Centres for Disease Control and Prevention, Tianjin 300011, China

${ }^{e}$ School of Agriculture, South China Agricultural University, Guangzhou 510642, China

${ }^{f}$ Guangdong Key Laboratory of Nanomedicine, Shenzhen Engineering Laboratory of Nanomedicine and Nanoformulations, CAS Key Laboratory of Health Informatics, Institute of Biomedicine and Biotechnology, Shenzhen Institutes of Advanced Technology, Chinese Academy of Sciences, Shenzhen 518055, China.

E-mail: pf.zhang@siat.ac.cn

$\dagger$ Electronic supplementary information (ESI) available: The PL intensity of TTAPE at different concentrations, stability of visual discrimination, and the influence of TTAPE on the PCR process. See DOI: 10.1039/c9qm00279k
}

vigorous supervision of genetically modified foods and other related documents has been promulgated. For GMO inspection and their environmental risk assessment, simple, effective and inexpensive on-field tests for GM events and/or GM contents are urgent to be developed. Various methodologies employed to analyze and/or detect the presence of GMO in food products have been reported, such as the polymerase chain reaction (PCR), ${ }^{3}$ enzyme linked immunosorbent assays (ELISA), ${ }^{4}$ microarrays, ${ }^{5}$ ${ }^{1} \mathrm{H}$ NMR, ${ }^{6}$ mass spectrometry, ${ }^{7}$ near infrared spectrometry (NIR), etc. $^{8,9}$ The detection methods for GMO presence in food are versatile. However, most of them need to be performed in a laboratory. The consequences of relying on the slow central laboratory approach are that long time period is needed before the lab results are made available. An ideal identification technique should be rapid, easy to use and most importantly, suitable for on-site analysis.

PCR methods are the most common and generally accepted detection methods for identifying the presence of GMO. ${ }^{10}$ These methods generally need to amplify the transgene with known sequences and compare the amplified fragment with that of the corresponding reference gene to obtain reliable results. The reported methods are sensitive, qualitative or quantitative, specific and precise, but there are also some disadvantages including high cost, difficulty to use, special needs, long duration, and so on. ${ }^{11}$ One solution was the application of an affordable, rapid, and easy to use portable PCR device to perform rapid DNA amplification. ${ }^{12}$ For identification of amplified products, agarose 
gel electrophoresis, which separates DNA fragments based on their size differences, is generally used. Some new DNA detection techniques with the advantages of high throughput, better specificity, and higher resolution have been developed in addition to agarose gel electrophoresis. However, they require expensive instruments with special software, such as a real-time PCR machine, microarray scanner, and capillary electrophoresis apparatus, etc. The visual detection of DNA amplification results is much more convenient for on-site analysis. ${ }^{13}$

A new class of fluorescent materials with aggregationinduced emission (AIE) properties have been developed by Tang's group since 2001. ${ }^{14}$ Opposite to the photophysical phenomenon of aggregation caused quenching (ACQ) dyes, AIEgens are almost non-emissive in the molecularly dissolved state but emit intensely in the aggregated state due to the restriction of intramolecular motions and prohibition of energy dissipation via non-radiative decay channels. This unique property made easy construction of light-up/turn-on bioprobes possible by taking advantage of the AIE process. Under appropriate conditions, an AIE-based fluorescent probe luminesces dimly with very low background noise. Once it is interacted with target bioanalytes, the intramolecular motions of the AIEgen are restricted, and the lighted-up fluorescence signal can thereby be detected in a sensitive manner. Furthermore, bioprobes based on AIEgens can visualize the organelles, cells, bacteria, tissues, etc., with high contrast and superb spatial resolution and without the need for washing. ${ }^{15-20}$ However, to the best of our knowledge, there have been few reports on GMO detection based on AIEgens. A series of cationic TPE derivatives with responsive ability to light up the negatively charged nucleic acids with a complex 3D structure in solution have been developed, which inspired us to develop a visual detection approach for GMO detection. In this work, we developed an "ALL in ONE" GMO visual discrimination system through the combination of a portable device (PCR, centrifuge, spectrometer, etc.) and DNA light-up AIEgens (1,1,2,2-tetrakis[4(2-bromo-ethoxy)phenyl]ethene, TTAPE). ${ }^{21-23}$ It is called "ALL in ONE" because all portable equipment could be packed in one bag, and all transgenic food discrimination experiments could be carried out on the field with the help of portable power. It should be pointed out that it is the first attempt using AIE materials for on-site GMO detection.

\section{Results and discussion}

\section{Principles}

As shown in Scheme 1, the "ALL in ONE" GMO visual discrimination system consists of a genomic extraction kit, detection kits, portable power supply, portable centrifuge, portable PCR device, portable spectrometer, portable storage dewar, portable computer, and a mortar and pestle. TTAPE (the mass spectrometry results could be found in Fig. S1, ESI $\dagger$ ) showed the best performance towards a guanine-rich DNA strand (e.g., G-quadruplex structure formed by human telomeric DNA sequences) via electrostatic attraction due to the perfect structural match. Therefore, it is

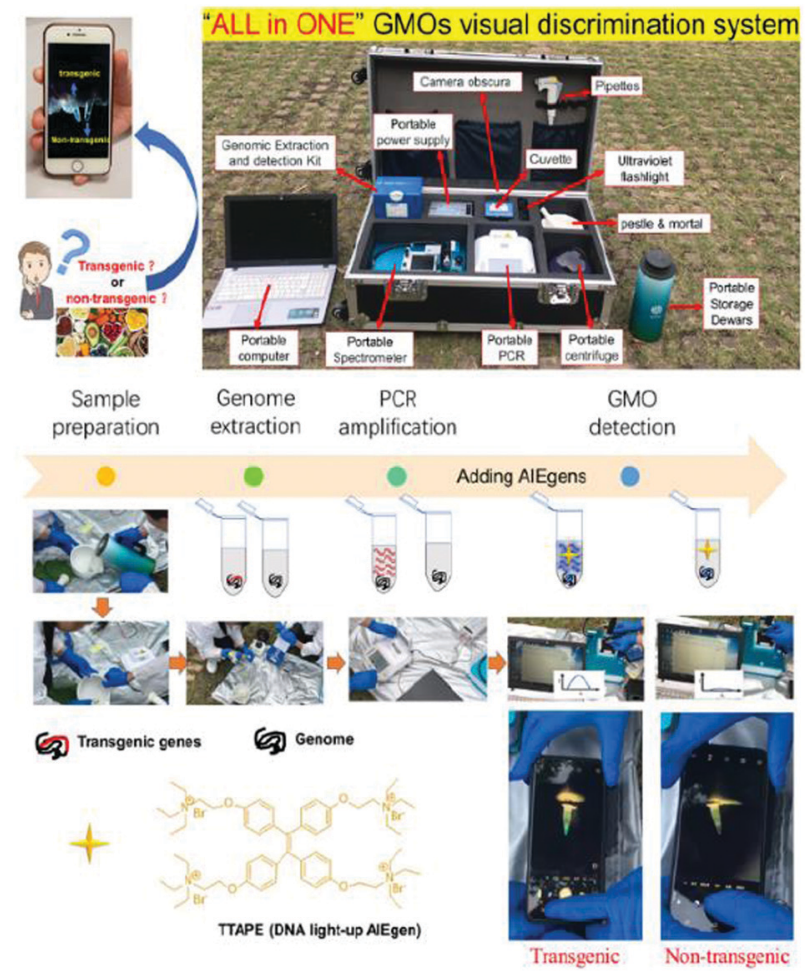

Scheme 1 Scheme for visual discrimination of transgenic and non-transgenic food using DNA-binding AlEgens and a brief introduction of the "ALL in ONE" GMO portable detection system. The portable detection system consisted of the following parts: genomic extraction and detection kit (genomic extraction reagents, mini-column, PCR primers, AlEgens, etc.); portable power supply; camera obscura (for visual detection); cuvette; pipettes; ultraviolet flashlight (for visual detection); mortar and pestle (for sample preparation); portable storage dewars (for sample preparation); portable centrifuge (for sample preparation); portable PCR device (for genomic sequence replication and amplification); portable spectrometer (for wavelength detection); portable computer (for data acquiring and analysis).

reasonable to anticipate that the direct visualization of transgenic food may be enabled by using TTAPE in combination with the PCR. The whole experiment could be carried out in the field. Firstly, the DNA was extracted from the samples. Different from the non-transgenic samples, there are transgene sequences in the genome of transgenic samples. The number of transgene fragments is exponentially amplified in PCR cycles upon the addition of the corresponding primer, which is further stained with TTAPE. When TTAPE is electrostatically bound to a DNA strand (G1), its intramolecular rotation is restricted, and a bright emission can be activated.

Due to the large amounts of PCR products, the fluorescence from the sample can be directly observed by the naked eye or recorded by a smartphone. In contrast, the emission was very low and could not be clearly visualized for the non-transgenic sample. The UV-vis spectral changes of AIEgen before and after aggregation were also measured and the results showed only small changes (Fig. S2, ESI $\dagger$ ).

As a proof of concept, the PL (photo-luminescence) intensities of the transgenic and non-transgenic samples were obtained and 

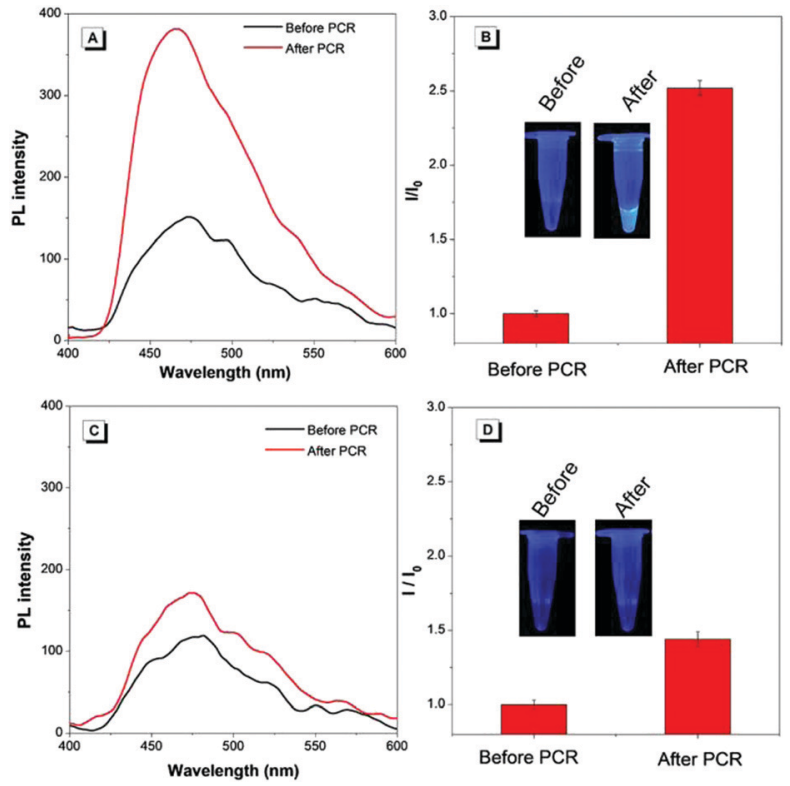

Fig. 1 Detection of transgenic material based on PCR amplification and TTAPE. (A) PL spectra of transgenic samples. (B) The maximum PL intensity ratio of transgenic samples after and before the PCR $\left(/ / I_{0}\right)$; inset: visual photos. (C) PL spectra of the non-transgenic sample. (D) The maximum PL intensity ratio of non-transgenic samples after and before the PCR $\left(I / I_{0}\right)$; inset: visual photos obtained with a smartphone. The background was due to the autofluorescence of the Eppendorf (EP) tube under UV irradiation, not from AlEgens.

compared before and after the PCR, respectively. As shown in Fig. $1 \mathrm{~A}$ and $\mathrm{B}$, for the transgenic sample, the PL intensity increased about 2.5 times after the PCR. Hence visual discrimination can be clearly made. However, the PL intensity of the nontransgenic sample showed only a small change after the PCR, which could not be discriminated visually (Fig. 1C and D). The results showed that the visual method was specific for transgenes.

\section{Method optimization}

The effect of the number of PCR cycles and TTAPE concentration on the performance of our method was studied. It's known that the amount of DNA increases exponentially during the PCR process. ${ }^{24}$ As a result, the emission was enhanced with the increase in the amount of DNA combined with TTAPE. Along with the increase in the number of PCR cycles (with 5 cycles as increments), the relative intensity increased (Fig. 2). The emission intensity was also enhanced with the increase in the amount of TTAPE. To make a compromise between analysis time and final visual results, $500 \mu \mathrm{M}$ TTAPE was taken as the final concentration, and 25 PCR cycles were adopted. We have conducted an interference experiment. The results showed that the negatively charged compounds interfere little with detection. They can interact with positively charged TTAPE, but the emission was low (Fig. S3, ESI $\dagger$ ). The visual discrimination can only be achieved with a large number of genes copies (long chain DNA) produced after the PCR process. As shown in Fig. S4 (ESI $\dagger$ ), the PL intensity increased sharply from 0 to $400 \mu \mathrm{M}$ and kept increasing between $400 \mu \mathrm{M}$ to $1500 \mu \mathrm{M}$. The images taken

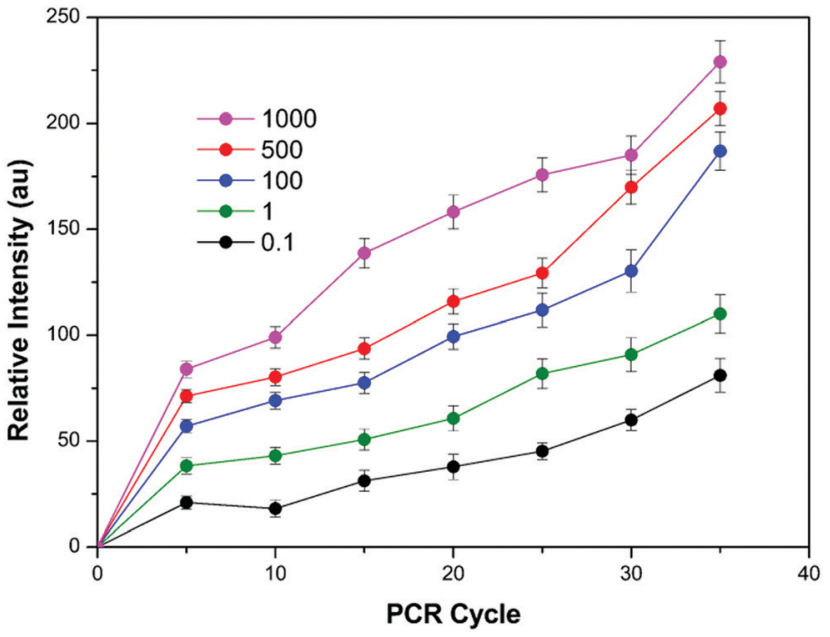

Fig. 2 The relative intensity under different PCR cycles and different concentrations of TTAPE. The results were obtained with the portable spectrometer.

by the camera in the Gel Imaging System were consistent with fluorescence measurement data (inset). Besides, the stability of visual discrimination results under white light was also evaluated. It showed that the discrimination results can be stable for longer than $48 \mathrm{~h}$ under white light (Fig. S5, ESI $\dagger$ ). The results confirmed that our proposed method is very stable for real sample analysis. As one of the water-soluble DNA-binding AIEgens, TTAPE showed high stability and shortened the number of PCR cycles due to the typical aggregation induced emission behaviors. Compared to existing DNA-binding dyes with an ACQ effect, which could be used only in dilute solutions, TTAPE showed better performance (Fig. S6, ESI $\dagger$ ).

We further evaluated the influence of the addition of TTAPE on the PCR process, which had little effect (Fig. S7, ESI $\dagger$ ). It is ascribed that the restriction induced emission of TTAPE was driven by the electrostatic interaction between the positive ammonium group in TTAPE and the negative phosphate backbones of DNA. In contrast, the PCR process might be eliminated for the other nucleic acid dyes inserting themselves into the spaces between the base pairs of the double helix. ${ }^{25}$ Therefore, TTAPE showed much more potential in the real-time PCR process.

\section{Method validation}

To further evaluate the versatility of our method, two more samples with different transgenic sequences were tested. As seen in Fig. 3, the three kinds of transgenic samples can be visually discriminated, which was validated by agarose gel electrophoresis. A DNA fragment of 369 bp was found in papaya, and a DNA fragment of $333 \mathrm{bp}$ for transgenes in corns and soybeans. The proposed method showed great potential for in situ and on-site discrimination of transgenic samples in comparison to conventional agarose gel electrophoresis with the aid of a portable PCR device. In addition, we have conducted a limit of detection assay for contaminated seeds to define the limit of the lowest proportion of GM contamination that this method could detect. As shown in Fig. S8 (ESI $\dagger$ ), even if only $0.01 \mathrm{wt} \%$ of the transgenic 

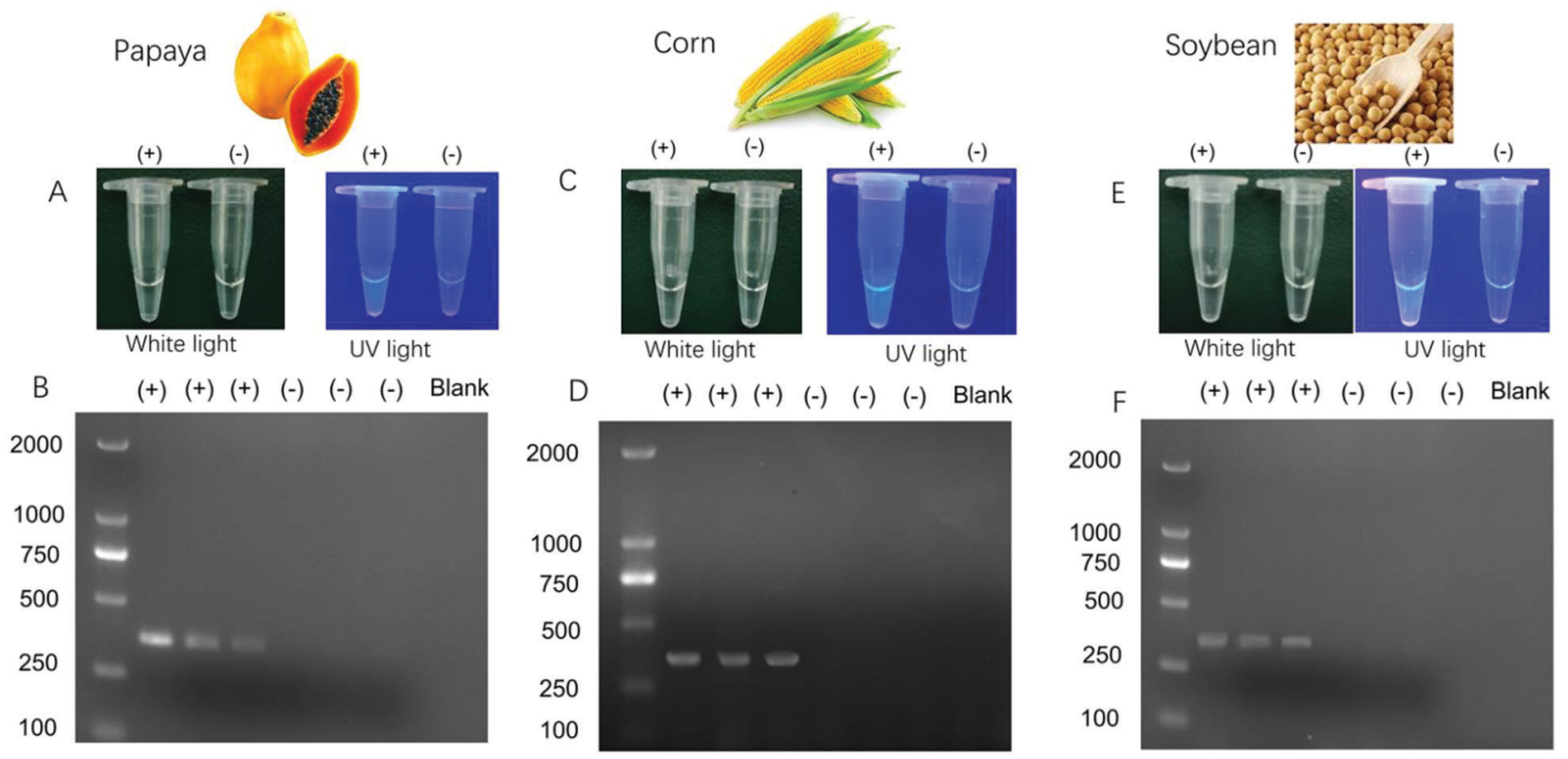

Fig. 3 The visual discrimination results by DNA-binding AlEgens (,$C$ and E) and results after agarose gel electrophoresis for transgenic and non-transgenic papaya ( $A$ and $B$ ), corn (C and D) and soybean (E and F). " +" represents three parallel transgenic samples, "-" represents three parallel non-transgenic samples. The expected fragments for papaya, corn and soybean were $369 \mathrm{bp}, 333 \mathrm{bp}$, and 333 bp respectively.

sample is mixed in the real sample, it could still be detected using our proposed method.

\section{Conclusions}

A convenient on-site and visual discrimination method for transgenic food was developed based on water-soluble DNAbinding AIEgens. The results indicated that the visual method was specific for transgenes. The discrimination can be stable as long as $48 \mathrm{~h}$ under white light. With the aid of a small-scale, portable PCR instrument, it's convenient to apply this method for rapid and on-site analysis. Given its simplicity, easy operation, sensitivity, and cost effectiveness, this method can be extended to diagnosis of genetic diseases and infection monitoring in relevant studies. The ALL in ONE system could be further miniaturized through the integration of the PCR device, spectrometer and AIEgens to accomplish point-of-care detection of GMO. More importantly, it should be pointed out that this is the first attempt using AIEgens along with portable devices to develop an on-site visual discrimination approach. It is a fact that the detection time seemed to be a little long ( $\sim 2$ hours); however, we believe that the detection time could be reduced by integration with a microfluidic system in which the whole process can take place. We are trying to develop more on-site visual discrimination approaches based on AIEgens with various responsive abilities and push AIEgens to real analytical applications.

\section{Experimental section}

\section{Materials and methods}

Transgenic papayas were kindly provided by South China Agricultural University. Transgenic corn and beans were kindly provided by Tianjin Centres for Disease Control and Prevention. The EZ-10 Spin Column Plant Genomic DNA Purification Kit (50 PREPS, No. B518261), Taq PCR Mix (2X, without dye, No. B639293), 6X Glycerol Gel Loading Buffer II (with Xylene Cyanol, BPB, Tris-HCl, EDTA) (No. B548314) and DNA Marker D (250 PREPS, No. B600335) were purchased from Sangon Biotech (Shanghai, China). The primers for Rep genes in transgenic papaya are REP-F: 5'-CTTTGGTGCGGAAAAGTTGT-3', REP-R: $5^{\prime}$-CCATAGCCCACAGTCGAATT $-3^{\prime}{ }^{7}{ }^{7}$ the primers for CP4-epsps in transgenic corn and transgenic soybean are MCP4ES-F: 5'-ACGGTGAYCGTCTTCCMGTTAC-3', mCP4ES-R: 5'-GAACAAGCARGGCMGCAACCA- $3^{\prime}{ }^{26}$ They were also purchased from Sangon Biotech (Shanghai, China). The PCR thermocycling process was carried out in the field using a portable thermocycling system (Leopard MINI PCR, Leopard scientific instruments Co. Ltd, China). The fluorescence of the samples was measured using a portable fluorescence measurement system (LIFS-405, Guangzhou BiaoQi Optoelectronics Technology Development Co., Ltd). The details of the portable detection system could be found in Scheme 1.

\section{Extraction of genomic DNA and PCR conditions}

$100 \mathrm{mg}$ of the sample was ground in liquid nitrogen using a mortar and pestle. DNA was extracted following the manufacturer's specifications with minor modifications. Briefly, the buffer PCB and $\beta$-mercaptoethanol were added and vibrated for $25 \mathrm{~min}$, then chloroform was added and the upper layer was transferred to a clean centrifuge tube. Then buffer and ethanol were added and transferred to the Ezup column. The column was washed twice with wash solution, and TE buffer was finally added to centrifuge the mixture for 2 min to obtain the final genomic DNA. The PCR was performed with a portable thermocycling system. For the 
transgenes in papaya, all PCR procedures were performed in a final volume of $30 \mu \mathrm{L}$ with the following reagent concentrations: genomic DNA $120 \mathrm{ng}$, primer mix of REP F and REP R $(6 \mu \mathrm{L}$, $10 \mu \mathrm{M}$ ), Taq PCR Mix $15 \mu \mathrm{L}$. Thermal cycler conditions were as follows: initial denaturation at $95{ }^{\circ} \mathrm{C}$ for $3 \mathrm{~min} ; 25$ cycles consisting of dsDNA denaturation at $94{ }^{\circ} \mathrm{C}$ for $30 \mathrm{~s}$; primer annealing at $57^{\circ} \mathrm{C}$ for $30 \mathrm{~s}$; primer extension at $72{ }^{\circ} \mathrm{C}$ for $60 \mathrm{~s}$; and final elongation at $72{ }^{\circ} \mathrm{C}$ for $7 \mathrm{~min}$. For the transgenes in soybean and corn, PCR procedures were performed in a final volume of $30 \mu \mathrm{L}$ with the following reagent concentrations: genomic DNA $120 \mathrm{ng}$, primer mix of mCP4ES-F and mCP4ES-R $(6 \mu \mathrm{L}, 10 \mu \mathrm{M})$, Taq PCR Mix $15 \mu \mathrm{L}$. Thermal cycler conditions were as follows: initial denaturation at $94{ }^{\circ} \mathrm{C}$ for $3 \mathrm{~min} ; 25$ cycles consisting of dsDNA denaturation at $94{ }^{\circ} \mathrm{C}$ for $30 \mathrm{~s}$; primer annealing at $62{ }^{\circ} \mathrm{C}$ for $30 \mathrm{~s}$; primer extension at $72{ }^{\circ} \mathrm{C}$ for $60 \mathrm{~s}$; and final elongation at $72{ }^{\circ} \mathrm{C}$ for $5 \mathrm{~min}$. TTAPE at a concentration of $500 \mu \mathrm{M}$ was added following the PCR.

\section{Agarose gel electrophoresis}

PCR products were analyzed using agarose gel electrophoresis (Beijing Liuyi biotechnology Co. Ltd, China). The gel was prepared with $2.0 \%$ of agarose in Tris Borate EDTA (TBE). $100 \mu \mathrm{M}$ of ethidium bromide (EtBr) was used. The running conditions were constant voltage at $100 \mathrm{~V}$ for $50 \mathrm{~min}$ in TBE. The image was taken using a Tanon 1600/1600R Gel Imaging System (Shanghai, China).

\section{Conflicts of interest}

There are no conflicts to declare.

\section{Acknowledgements}

This work was supported by the Science and Technology Planning Project of Guangdong Province of China (2018B020208005), the National Natural Science Foundation of Guangdong Province of China (2016A030313136) and the Guangdong Provincial Key Platform and Major Scientific Research Projects for Colleges and Universities (2015KCXTD029). The authors would like to acknowledge Prof. Dr Ryan T. K. Kwok for his suggestions on manuscript writing. We also acknowledge the technical support received from Thonebio. Co. Ltd, AIEgen Biotech Co., Ltd, and Mr Tianwei Chu from Guangzhou BiaoQi Optoelectronics Technology Development Co., Ltd. The authors would like to acknowledge Dr Yahui Zhang for her contribution on the figure artwork.

\section{Notes and references}

1 H. A. Kuiper, G. A. Kleter, H. P. J. M. Noteborn and E. J. Kok, Plant J., 2001, 27, 503.

2 A. König, A. Cockburn, R. W. R. Crevel, E. Debruyne, R. Grafstroem, U. Hammerling, I. Kimber, I. Knudsen, H. A. Kuiper, A. A. C. M. Peijnenburg, A. H. Penninks, M. Poulsen, M. Schauzu and J. M. Wal, Food Chem. Toxicol., 2004, 42, 1047.
3 K. Rudi, I. Rud and A. Holck, Nucleic Acids Res., 2003, 31, e62.

4 G. Liu, W. Su, Q. Xu, M. Long, J. Zhou and S. Song, Food Control, 2004, 15, 303-306.

5 R. Bordoni, A. Germini, A. Mezzelani, R. Marchelli and G. D. Bellis, J. Agric. Food Chem., 2005, 53, 912.

6 Z. Jiao, X. X. Si, Z. M. Zhang, G. K. Li and Z. W. Cai, Food Chem., 2012, 135, 285.

7 Z. Jiao, J. C. Deng, G. K. Li, Z. M. Zhang and Z. W. Cai, J. Food Compos. Anal., 2010, 23, 640-647.

8 L. J. Xie, Y. B. Ying, T. J. Ying, H. Y. Yu and X. P. Fu, Anal. Chim. Acta, 2007, 584, 379.

9 Z. Jiao, X. X. Si, G. K. Li, Z. M. Zhang and X. P. Xu, J. Agric. Food Chem., 2010, 58, 1746.

10 Y. Zhao, F. Chen, Q. Li, L. Wang and C. Fan, Chem. Rev., 2015, 115, 12491.

11 W. Xu, L. Xie, Z. Ye, W. Gao, Y. Yao, M. Chen, J. Qin and Y. Ying, Sci. Rep., 2015, 5, 11115.

12 P. Belgrader, S. Young, B. Yuan, M. Primeau, L. A. Christel, F. Pourahmadi and M. A. A. Northrup, Anal. Chem., 2001, 73, 286.

13 Y. Cao, K. Zheng, J. Jiang, J. Wu, F. Shi, X. Song and Y. Jiang, Food Chem., 2018, 266, 73.

14 J. D. Luo, Z. L. Xie, J. W. Y. Lam, L. Cheng, H. Y. Chen, C. F. Qiu, H. S. Kwok, X. W. Zhan, Y. Q. Liu, D. B. Zhu and B. Z. Tang, Chem. Commun., 2001, 1740.

15 J. Mei, N. L. C. Leung, R. T. K. Kwok, J. W. Y. Lam and B. Z. Tang, Chem. Rev., 2015, 115, 11718.

16 J. Liang, B. Z. Tang and B. Liu, Chem. Soc. Rev., 2015, 44, 2798.

17 J. Mei, Y. H. Huang and H. Tian, ACS Appl. Mater. Interfaces, 2018, 10, 12217.

18 M. Gao and B. Z. Tang, ACS Sens., 2017, 2, 1382.

19 X. G. Gu, R. T. K. Kwok, J. W. Y. Lam and B. Z. Tang, Biomaterials, 2017, 146, 115.

20 J. Qian and B. Z. Tang, Chem. Rev., 2017, 3, 56.

21 Y. N. Hong, M. Häußbler, J. W. Y. Lam, Z. Li, K. K. Sin, Y. Q. Dong, H. Tong, J. Z. Liu, A. J. Qin, R. Renneberg and B. Z. Tang, Chem. - Eur. J., 2008, 14, 6428.

22 M. Wang, D. Q. Zhang, G. X. Zhang, Y. L. Tang, S. Wang and D. B. Zhu, Anal. Chem., 2008, 80, 6443.

23 X. D. Lou, Y. Zhuang, X. L. Zuo, Y. M. Jia, Y. N. Hong, X. H. Min, Z. Y. Zhang, X. M. Xu, N. N. Liu, F. Xia and B. Z. Tang, Anal. Chem., 2015, 87, 6822.

24 J. Li, T. Deng, X. Chu, R. Yang, J. Jiang, G. Shen and R. Yu, Anal. Chem., 2010, 82, 2811.

25 S. Sindbert, S. Kalinin, H. Nguyen, A. Kienzler, L. Clima, W. Bannwarth and C. A. Seidel, J. Am. Chem. Soc., 2011, 133, 2463.

26 Detection of genetically modified plants and derived products-qualitative PCR method for CP4-epsps gene. No. 1861 Announcement of the Ministry of Agriculture of the People's Republic of China. 